Cita bibliográfica: Esquivel Ríos, R. y López Molina, L.A. (2018). Los “usos y costumbres" del estado de Oaxaca en las empresas turísticas de Bahías de Huatulco. Una caracterización de la cultura organizacional de Hofstede. Investigaciones Turísticas (15), pp. 128-146. http://dx.doi.org/10.14198/INTURI2018.15.06

\title{
Los "usos y costumbres" del estado de Oaxaca en las empresas turísticas de Bahías de Huatulco. Una caracterización de la cultura organizacional de Hofstede
}

\section{The "uses and customs" of the state of Oaxaca in the tourist companies of Bahías de Huatulco. A characterization of Hofstede's organizational culture}

Rocío Esquivel Ríos. Universidad del Mar, México. chioesquivel@hotmail.com Luis Alberto López Molina. Universidad del Mar, México.7uis.Ipz@gmail.com

\section{RESUMEN}

El presente trabajo tiene como finalidad el estudio de la cultura organizacional de los colaboradores de las empresas turísticas del CIP Bahías Huatulco a partir de una de las plataformas desarrolladas por Hofstede, atendiendo particularmente a la práctica de "usos y costumbres" que se tienen en la región. A partir de aquí el artículo propone identificar la plataforma correspondiente a la orientación a largo plazo del destino en general y determinar cuál es la importancia que se le otorga a los "usos y costumbres". Lo anterior se llevará a cabo mediante una encuesta realizada a los colaboradores de las empresas turísticas del destino en cuestión, y posteriormente el análisis de la información a partir de la estadística descriptiva. Finalmente se concluye con la importancia que estas prácticas culturales tienen dentro de la actividad laboral de los colaboradores.

El artículo se desarrolla en cuatro apartados generales, comenzando por el estado del arte que aborda los principales tópicos para el estudio de la cultura organizacional. En el siguiente apartado se observa la delimitación geográfica del contexto de estudio, se continúa con el desarrollo metodológico de la investigación y los resultados obtenidos. Por último, las reflexiones apuntan a la relevancia de los "usos y costumbres" en la gente originaria de la región y la visión u orientación que se tiene de acuerdo con la teoría de Hofstede.

Palabras clave: usos y costumbres, cultura organizacional, empresas turísticas

\section{ABSTRACT}

This study seeks to analyze the organizational culture of the collaborators of the tourist companies of the CIP Bahías Huatulco based on one of the platforms developed by Hofstede, with particular focus on the practice of customs and traditions in the region. The article identifies the platform corresponding to the long-term direction of the destination in general and 
determines the importance given to customs and traditions. This will be carried out by means of a survey conducted among the collaborators of the tourist companies of the destination in question and the subsequent analysis of the information drawn from the descriptive statistics. Finally, it will discuss the importance that these cultural practices have within the work activity of employees. The article is divided into four main sections, beginning with the development of the conceptual context that addresses the main topics for the study of organizational culture. The following section addresses the geographical delimitation of the place of study. The methodological development of the research and the results obtained are described in the third section. Finally, the concluding reflections point to the relevance of the customs and traditions for the people native to the region and the vision or orientation in accordance with Geert Hofstede's theory.

Keywords: Customs and traditions, organizational culture, tourism companies

\section{INTRODUCCIÓN}

Geert Hofstede (1984) antropólogo y escritor holandés, realizó un estudio de la cultura organizacional durante dos periodos, en 1968 y 1972, a través de 72 filiales de IBM en 53 países diferentes, con la idea de obtener un mejoramiento de las relaciones humanas desde una perspectiva global.

El objetivo de estudiar la cultura organizacional radica en la creencia de concebir a la cultura como la parte invisible de una organización que está constituida por valores compartidos con la mayoría de sus habitantes; quienes determinan normas de convivencia y en gran medida las soluciones políticas y organizativas que son observadas de forma macro y micro en cada país.

Bajo el argumento anterior, Hofstede (1984) creó cinco plataformas, las cuales corresponden a los parámetros para medir la cultura. La última de ellas corresponde a la orientación a largo plazo, en el que se aprecia una sociedad con visión a largo plazo que tiende a priorizar la capacidad de ahorrar y perseverar, mientras que los valores asociados con la orientación a corto plazo son el respeto por las tradiciones, las obligaciones sociales y la protección de la reputación propia.

Para el desarrollo de esta investigación y, particularmente de la plataforma mencionada, así como de los "usos y costumbres", es importante identificar algunas diferencias existentes en las definiciones de cultura. Por ejemplo, Méndez (2003) define a la cultura organizacional como la conciencia colectiva que se expresa en el sistema de significados compartidos por los miembros de la organización, que los identifica y diferencia de otros al mismo tiempo, institucionalizando y estandarizando sus conductas sociales.

Por otra parte, una definición antropológica de la cultura es el conjunto de los procesos sociales de significación, o de un modo más complejo la cultura abarca el conjunto de procesos sociales de producción, circulación y consumo de la significación en la vida social (García, 2004). 
Una definición sociológica de la cultura es la que aportan Vera y Rodríguez (2009) quienes mencionan que la cultura es un sistema de interrelaciones entre los procesos individuales ontogenéticos, los sociales e históricos del comportamiento colectivo en un corte de tiempo, y los antropológicos e históricos que hacen posible los productos culturales, incluyendo a las manifestaciones artísticas, cotidianas, científicas, tecnológicas y las del tipo folklórico.

Las definiciones anteriores forman parte del contexto de estudio, de tal forma que enmarcan los "usos y costumbres", término que se desarrollará más adelante. El objetivo planteado para este trabajo es identificar la importancia de los "usos y costumbres" de los colaboradores de las empresas turísticas de Bahías de Huatulco, a partir de la plataforma visión a largo plazo desarrollada por Hofstede (1984). Lo innovador de este trabajo es que permite visibilizar los aspectos a corto y largo plazo de Hofstede, enfatizando la cuestión de los "usos y costumbres" como una forma de organización social que se refleja en el aspecto laboral, específicamente en las empresas turísticas de Bahías de Huatulco. En ese sentido y siguiendo el lineamiento anterior se desarrolla el siguiente texto.

\section{MARCO CONCEPTUAL}

\subsection{La cultura y los "usos y costumbres"}

Es importante reconocer que en cualquier clase de turismo hay siempre un encuentro con la cultura receptora, es decir, el contexto en el que se desenvuelve la actividad turística. Lo anterior hace que la cultura como los bienes materiales e inmateriales, los "usos y costumbres", la gastronomía, los ritos y la vida cotidiana "se perfilen como activos que pueden contribuir al desarrollo socioeconómico de comunidades, empresas o individuos" (Hernán, 2006, p.1). Aunque el autor apuesta por los bienes materiales e inmateriales como fines para la actividad turística, lo que se rescata de este planteamiento es su potencial cultural.

El uso del término "usos y costumbres" es muy generalizado; en un primer momento describe "un sistema cultural propio" (Canedo, 2008, p.403). Se entiende que esta categoría puede abarcar distintos ámbitos como: la medicina tradicional, los ritos, las fiestas, etc. No obstante, puede restringirse a cuatro espacios diferentes sobre los modos propios de vida de la gente: a) lo político, b) lo económico, c) lo social y d) lo cultural. Sin embargo, termina siendo una palabra de uso común y general.

Los "usos y costumbres" Nahmad los define como "los modos de vida de la gente; es la forma de reproducción social que tienen los pueblos" (citado en Canedo, 2008, p.404). En Oaxaca es común referirse y emplear el término "usos y costumbres". Sin embargo, éste mayormente se ha desarrollado en el aspecto político. Es decir, en la forma en que distintos municipios y localidades llevan a cabo su vida política y la elección de sus gobernantes. En la ciencia antropológica a este aspecto se le reconoce con el sistema de cargos, tanto el civil como el religioso.

Para el presente texto, los "usos y costumbres" son considerados como un modo de vida ligados a una cosmovisión, en el que la transmisión de conocimientos es indispensable para la reproducción social. Se destacan los aspectos social y cultural, ya que para el presente 
estudio los "usos y costumbres" se ven reflejados en el trabajo que desempeñan los empleados de las empresas turísticas en Bahías de Huatulco.

Por otra parte, Hosftede (1984) menciona que la cultura es una parte invisible (de una organización) que está integrada por valores compartidos por la mayoría de sus habitantes. Convertidos en normas de convivencia, determinaban en gran medida las soluciones políticas y organizativas que son observadas de forma macro y micro en cada país.

En ese sentido, los valores, según el propio autor, conforman el corazón de la cultura organizacional. Por ello, Hofstede (1984, p.15) menciona que los programas mentales colectivos consisten en ciertos patrones estables de pensamiento, sentimiento y acción, que hacen posible la distinción de un grupo social de otro. De este modo, clasifica a las manifestaciones culturales en cuatro categorías: símbolos, héroes, rituales y valores. Hofstede (1984) agrupa a los tres primeros como prácticas porque son visibles aunque sus significados dependen de la percepción de los miembros de la sociedad en la que se encuentran.

Por otro lado, los símbolos se refieren a las palabras, gestos u objetos que cuentan con un significado propio dentro de la cultura. Los héroes son personas reales o que nos sirven como ejemplo de conducta para los miembros de la sociedad. Y por último los rituales, los cuales son actividades sociales de mucha relevancia.

\subsection{Importancia del Capital Humano}

Hoy en día el término capital humano es muy utilizado para hacer referencia a los trabajadores de una empresa. Sin embargo, este término desarrollado en la década de los 60's abarca mucho más que la idea anterior. Schultz (1961) y Becker (1964) mencionan que el capital humano se relaciona con la productividad y es definido como la suma de las inversiones en educación, formación en el trabajo, emigración o salud, que tienen como consecuencia un aumento en la productividad de los trabajadores.

Boisier (2002) (citado en Navarro, 2005) menciona que el capital humano corresponde al valor que generan las capacidades de las personas mediante la educación, la experiencia, la capacidad de conocer, de perfeccionarse, de tomar decisiones y relacionarse con los demás. Asimismo, propone considerar diversos tipos de capital, como son el capital cognitivo que corresponde a los conocimientos de los colaboradores; el simbólico, que se compone principalmente por el lenguaje; el cultural que se forma de las tradiciones, mitos y leyendas que se dan entre los colaboradores; el cívico que hace referencia a la confianza de las instituciones y; por último, el capital psicosocial en donde intervienen los sentimientos, ideas, deseos, sueños, etc.

Aunado a las definiciones anteriores, se han desarrollado algunas otras que tratan de explicar el funcionamiento de los colaboradores, coincidiendo en que este recurso es prioritario para el desarrollo y funcionamiento de la empresa. Además, se puede visualizar que el capital humano es mucho más que los trabajadores o empleados de cualquier organización, ya que es a través de su desempeño, desarrollo y el conjunto de las condiciones laborales que se reflejan en la productividad de la empresa. Siguiendo esta línea de ideas, se pueden identificar una gran variedad de condicionantes en el estudio del capital humano. 
Este término ha tomado tanta fuerza a lo largo de los años que hoy en día existen nuevas teorías sobre el capital humano como las de Gary Becker (1992) premio Nobel de Economía, o bien las teorías de los activos intangibles propuestas por Lev (2004). Becker (2002) menciona que la productividad de las economías modernas depende en gran medida de los que invierte en la adquisición de conocimientos y habilidades. Sin embargo, las estadísticas gubernamentales no incluyen los gastos en capital humano como ahorro o inversión. La educación, la capacitación laboral, el gasto en instituciones especializadas y en los servicios de salud contribuyen al capital humano, que es una imparte integral de las naciones. El mismo Becker (2002) menciona que diversos economistas estiman que el capital humano es responsable de más de la mitad de la riqueza de Estados Unidos y de otros países desarrollados.

Con lo anterior se puede entender la importancia que tiene el capital humano dentro de las empresas a nivel mundial. Para el caso de estudio no es diferente, el capital humano forma parte prioritaria del servicio ofrecido. Dentro de esta investigación se visualizó a un capital humano con características muy particulares que son inherentes a la región en la que se encuentran, como son los "usos y costumbres"; de lo que ya se ha abordado y que posteriormente se podrá identificar la relevancia que tienen en la conducta de los colaboradores. Para lograr lo anterior es necesario conocer el estudio de Hofstede, quien propone el análisis de la cultura organizacional a través de categorías, en las que se enmarcan las tradiciones y la cultura de los colaboradores.

\subsection{El estudio de Hofstede}

Esta investigación se desarrolla a partir de la construcción de las plataformas de Hofstede, utilizando este modelo para la evaluación de la plataforma denominada orientación a largo plazo. Hofstede (2001) demostró que hay agrupamientos culturales en el contexto regional y nacional que son muy persistentes en el tiempo y que afectan el comportamiento de las sociedades y de las organizaciones.

Tomando en consideración la afirmación anterior e implementándola en las empresas turísticas del CIP Bahías de Huatulco, se podría entender que los colaboradores dentro de sus organizaciones tienen una cultura arraigada por el contexto regional, social y cultural, que ha sido persistente a lo largo de los años. Entre ellas podrían destacar las que tienen que ver con los "usos y costumbres".

Para contextualizar a qué se refiere la orientación a largo plazo Chamorro (2016) describe el horizonte temporal de una cultura. Las sociedades con una alineación de corto plazo aprecian las tradiciones, el buen desarrollo de las relaciones entre sus integrantes, es decir, procuran tener organizaciones armoniosas y evitar el conflicto y la orientación a considerar el tiempo como una variable circular. Para esta clase de culturas u organizaciones el pasado y el presente son parte fundamental de su día a día. Por el contrario la orientación a largo plazo percibe el tiempo como una dimensión lineal y tiene mayor importancia el futuro que el pasado.

En el estudio de Hofstede (1972) se observa la visión que tienen los países hacia el futuro o bien hacia la conservación de las tradiciones, algunos de los resultados obtenidos durante dicha investigación son los que se muestran en la tabla siguiente. 
Gráfico 1. Orientación a largo plazo

\begin{tabular}{|c|c|c|}
\hline Orden & País & Índice de Orientación a Largo Plazo \\
\hline 1 & China & 118 \\
\hline 2 & Hong Kong & 96 \\
\hline 3 & Taiwán & 87 \\
\hline 4 & Japón & 80 \\
\hline 5 & Corea del sur & 75 \\
\hline 6 & Brasil & 65 \\
\hline 7 & India & 61 \\
\hline 8 & Tailandia & 56 \\
\hline 9 & Singapur & 48 \\
\hline 10 & Países bajos & 44 \\
\hline 11 & Bangladesh & 40 \\
\hline 12 & Suecia & 33 \\
\hline 13 & Polonia & 32 \\
\hline 14 & Alemania & 31 \\
\hline 15 & Australia & 31 \\
\hline 16 & Nueva Zelanda & 30 \\
\hline 17 & Estados Unidos & 29 \\
\hline 18 & Gran Bretaña & 25 \\
\hline 19 & Zimbabwe & 25 \\
\hline 20 & Canadá & 23 \\
\hline 21 & Filipinas & 19 \\
\hline 22 & Nigeria & 16 \\
\hline 23 & Paquistán & 00 \\
\hline
\end{tabular}

Fuente: Extraído de Vialadot (2008)

Se observa que China es considerado un país con orientación a largo plazo (con 118 puntos), aunque podría resultar paradójico ya que cuentan con historia, cultura y costumbres milenarias, sin embargo esta clase de países trabajan pensando en el futuro adaptando su pasado a la modernidad sin perder el respeto.

Por el contrario los países con orientación a corto plazo son Filipinas, Nigeria y Paquistán, recordando que estas culturas ponen énfasis en el pasado y la conservación de sus tradiciones, en este caso se puede pensar en la legendaria tradición religiosa de Filipinas o bien en los rituales emblemáticos de Nigeria.

Rozas y Sánchez (2004) mencionan que a mayor nivel de infraestructura, mayor nivel de orientación a largo plazo. Es decir una organización con un plan de dirección claro y políticas que regulen el manejo de la misma, dentro de las cuales se perciba enfrentar los retos presentes y estar encaminadas a tomar los retos que el futuro marca, teniendo en consideración su situación financiera y al mismo tiempo a los colaboradores.

Un ejemplo de sociedades con orientación a corto y largo plazo son los descritos por Herrasti (2013) en el que Japón es un país con una orientación a largo plazo y que su forma 
de vida se puede observar incluso en sus aulas de clases. En Japón, los estudiantes día con día preparan y estudian sus clases, realizan actividades que faciliten la comprensión de sus lecciones y previamente realizan lecturas que les facilitarán el entendimiento dentro de las aulas. Esta planeación de actividades en el presente trae consigo beneficios a futuro, ya que al momento de las evaluaciones, los alumnos se encuentran menos estresados, con más seguridad de sus conocimientos y, sobre todo, sin miedo a los resultados que puedan obtener. Es decir tienen una visión a largo plazo.

\subsection{Orientación a largo plazo}

Los valores de una sociedad con una visión a largo plazo son la capacidad de ahorrar y perseverar; los valores asociados con la orientación a corto plazo son el respetar la tradición, cumplir con obligaciones sociales y proteger la propia reputación.

Según Fang (2003) de acuerdo con Hofstede, la orientación a largo plazo se refiere a una cultura orientada al futuro, positiva, dinámica, basada en cuatro valores: perseverancia, ordenar las relaciones por estatus y observar ese orden, economía y tener sentido de vergüenza. Asimismo, Hofstede (2001) menciona que esta dimensión es un reflejo de la mentalidad hacia el futuro que tienen las sociedades, y por otro lado, refleja la mentalidad orientada hacia el pasado o las tradiciones.

De Mooij y Hofstede (2010) afirman que esta dimensión se refiere al grado o nivel, en que la sociedad tiene una perspectiva con enfoque al futuro, en lugar de tener un pensamiento a corto plazo, que es históricamente muy común. La orientación a largo plazo también se refiere a la importancia que las personas de una cultura planeen la vida a largo plazo, y por el contrario, la orientación a corto plazo tiene su enfoque en los valores que permiten a uno ser feliz en el momento.

Según Duarte y Zinder (2006) (citados por Guillén 2007, p. 41) un elemento motivacional para los miembros de equipos de proyectos enfocados a largo plazo es contribuir con los objetivos a largo plazo, tanto del proyecto como personales, entre ellos se encuentran la adquisición de conocimientos o destrezas. Individuos culturalmente orientados al corto plazo pueden volverse impacientes, especialmente en proyectos largos.

Bajo el contexto anterior se pueden mencionar que las culturas que tienen una orientación a largo plazo, son aquellas que dan importancia a la perseverancia y el ahorro, como la mayoría de países asiáticos. Los integrantes de esta sociedad esperan recibir frutos del presente posteriormente, por lo que la planeación es una pieza fundamental en sus organizaciones. Estas sociedades aprenden de las acciones del pasado, pero no viven en él, simplemente se preparan para vivir el futuro (Guillén, 2007).

Por su parte, aquellas organizaciones que ponen mayor valor al presente aprecian las acciones o hechos del pasado y tienen el interés de seguir conservando parte de esa historia a través de sus costumbres o tradiciones, las cuales siguen siendo trascendentes para ellos. Lo anterior sirve de base para entender el contexto teórico de la investigación, no obstante también es importante conocer el contexto espacial, el cual se desarrolla a continuación. 


\section{CONTEXTO DE ESTUDIO}

El proyecto de Bahías de Huatulco comenzó a realizarse en 1983 con un Plan Maestro pensado como el quinto desarrollo turístico integral de México, así como Ixtapa, Cancún, Los Cabos y Loreto (Salazar y Ramírez, 2003).

En ese sentido y de acuerdo con el Plan Maestro, el desarrollo se divide en tres etapas comprendidas entre 1984 y 2018. La primera (1984-1994) cubre las necesidades de urbanización presente y futura de tres bahías comunicadas entre sí por una avenida costera: Santa Cruz, Chahué y Tangolunda, además de la construcción de un aeropuerto internacional (Salazar y Ramírez, 2003).

En la segunda etapa el Plan Urbano de 1994 se definió, determinándose la ubicación de los hoteles a construir. Mientras más alejados se encuentren de las zonas aglomeradas su categoría debería aumentar y su densidad edificadora sería cada vez menor. Para la tercera etapa (2001) se construyó un muelle de cruceros en la Bahía de Santa Cruz, además de la organización de una agrupación ciudadana para la implantación de prácticas ambientales y la obtención de la certificación de Green Globe 21 (actualmente Earth Check).

Fue en 1984 que este territorio fue expropiado por el gobierno federal para construir el actual destino turístico conocido como Bahías de Huatulco, con la finalidad de impulsar el desarrollo regional económico, así como el de elevar la calidad de vida de la población de una de las entidades más pobres del país y más ricas en recursos naturales. En la actualidad, un alto porcentaje de la población residente en Huatulco proviene del interior del estado de Oaxaca e incluso de otros estados de la república, de tal forma, que ya se disputa entre locales y fuereños el rumbo político de esta comunidad González et al. (1997) (citados en el Programa de Manejo Parque Nacional Huatulco, CONANP, 2003).

Talledos (2012) menciona que la migración de estados vecinos como Chiapas y Guerrero ha sido consecuencia de la creación del CIP, ya que todos ellos fueron atraídos por el sector de la construcción al momento de desarrollar el destino y al mismo tiempo por los servicios turísticos, especialmente por las nuevas labores que la propia actividad turística demandaba.

Hablando concretamente de la ubicación geográfica, el CIP Bahías de Huatulco se localiza en el estado de Oaxaca, en el distrito de Pochutla, en el municipio de Santa María Huatulco, en el extremo suroeste del Golfo de Tehuantepec. Se ubica entre los $15.70^{\circ}$ y $15.78^{\circ}$ de latitud Norte y los $96.06^{\circ}$ y $96.20^{\circ}$ longitud Oeste. Cubre una extensión aproximadamente de $20 \mathrm{~km}$. de litoral. Algunas de sus bahías son Órgano y Maguey, Santa Cruz, Chahué, Tangolunda y Conejos. Por carretera se encuentra aproximadamente a $290 \mathrm{~km}$. de la ciudad de Oaxaca y a $750 \mathrm{~km}$. del Distrito Federal, también se tiene acceso al destino por vía área y marítima (FONATUR, 2006).

En 1984, como se ha dicho, comienza el Proyecto del CIP Bahías de Huatulco, mediante la solicitud de expropiación de la reserva territorial de una superficie de 21,000 ha., para destinarse al desarrollo turístico. El sitio contaría con $35 \mathrm{~km}$. de costa en los que se ubican 9 bahías y 36 playas. Dichos terrenos fueron puestos a disposición del Fondo Nacional de Fomento al turismo (FONATUR), con el fin de realizar las obras de infraestructura y equipamiento necesarias, de acuerdo con el Plan Maestro (Mendoza et al., 2011). 
De acuerdo con lo anterior la ubicación del CIP Huatulco y el desarrollo del mismo constituyen un factor de atracción tanto para nuevos inversionistas como para trabajadores potenciales y turistas. La convivencia entre estos dos grupos sociales y la propia comunidad receptora generan el nacimiento de fenómenos sociales de distintas índole, esta investigación se centra en el estudio de la comunidad receptora, quien en todo caso se podría ver afectada por la llegada de migrantes. A continuación se describe la metodología que se llevó a cabo durante la investigación.

\section{METODOLOGÍA}

Esta investigación se considera de carácter transeccional-exploratoria debido a que se tienen un conjunto de variables para una sola situación. Sampieri et al., (2006) mencionan que el propósito de los diseños transeccionales exploratorios es comenzar a conocer una variable o un conjunto de variables, una comunidad, un contexto, un evento, una situación.

En ese sentido, esta investigación se realizó conociendo una serie de variables que hacen referencia a la caracterización de una plataforma, lo cual da pie a una secuencia de investigaciones posteriores siguiendo la misma línea de estudio. En términos generales se trata de un trabajo exploratorio y de carácter descriptivo que incluyó a todo el universo de 72 sujetos de estudio, por lo que es una muestra no probabilística, un estudio de caso.

La clasificación del universo se llevó a cabo en dos grandes segmentos, instituciones públicas y privadas, dentro de los cuales se enlistaron las siguientes organizaciones:

- Empresas hoteleras

- Restaurantes

- Tours operadoras

- Servicios de transporte

- Instituciones reguladoras

- Universidades que brindan la carrera de turismo

Posterior a ello, para la identificación de las empresas se elaboró una matriz de coherencia en donde se identificaron los conceptos clave que midieron en su conjunto la visión a corto o largo plazo, según fuera el caso.

Gráfico 2. Matriz de coherencia

\begin{tabular}{|c|c|c|c|}
\hline CONCEPTOS & VARIABLES & INDICADORES & SUBINDICADORES \\
\hline \multirow{2}{*}{$\begin{array}{c}\text { Orientación a largo } \\
\text { plazo }\end{array}$} & Valores y virtudes & Largo plazo & Ahorro \\
\cline { 3 - 4 } & & Corto plazo & Perseverancia \\
\cline { 3 - 4 } & & Obligaciones sociales \\
\hline
\end{tabular}

Elaboración propia 
A partir de la tabla anterior se construyó una encuesta basada en preguntas enfocadas a identificar la importancia que los colaboradores tienen hacia el ahorro, la perseverancia, las tradiciones y las obligaciones sociales, que como ya se mencionó anteriormente, se reflejan en las organizaciones. Dicha encuesta contó con tres opciones de respuesta posibles, entabladas a partir de la escala de Likert.

El cuestionario constó de dos partes claramente definidas. En la primera, tras pedir al sujeto de investigación que defina su posición en la compañía (de mando o no), se recoge información relativa a la empresa en que labora; la segunda parte se dedica a recoger el punto de vista del entrevistado respecto a la visión a corto o largo plazo, según las prácticas realizadas. El vaciado de la información y la interpretación de la misma se hicieron a partir de gráficas para facilitar su comprensión, las cuales se detallan a continuación.

\section{RESULTADOS DEL CASO DE ESTUDIO}

\subsection{Ahorro}

En términos financieros el ahorro según Cotler (2003) es la diferencia entre el ingreso total y el gasto total, pero se debe considerar que el ahorro no solamente consiste en el dinero en efectivo; los bienes materiales también forman parte de éste, inclusive la materia prima también se constituye como un ahorro, hablando del caso de la siembra, en donde se espera recibir el fruto de la cosecha.

Esta variable dentro de la investigación apoya en la medición de las empresas que tienen una visión a largo plazo, recordando que el ahorro es una manera de prepararse para enfrentar el futuro. En ese sentido el cuestionamiento aborda si es que dentro de la empresa a la que pertenecen realizan prácticas de ahorro. Los resultados se muestran a continuación.

Gráfico 3. Ahorro

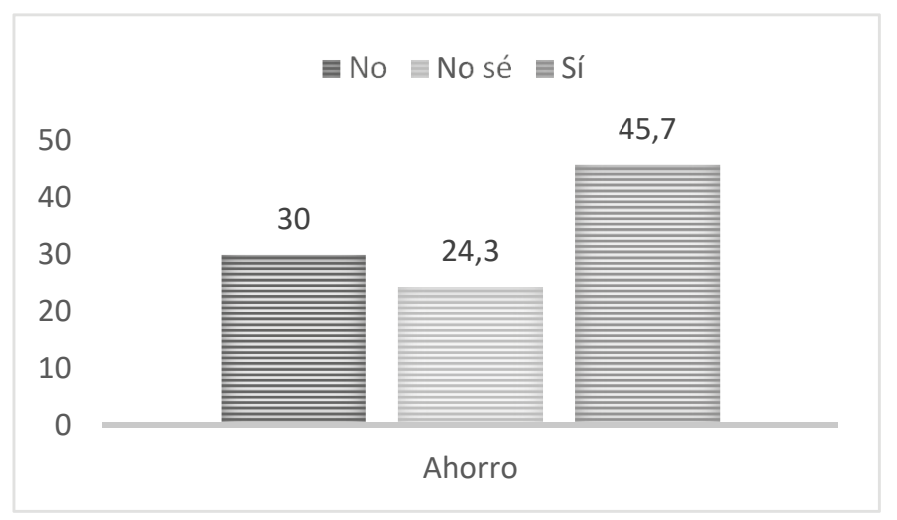

Fuente: elaboración propia con datos de la encuesta (30/04/2016)

En la gráfica se puede observar que los sujetos de estudio perciben tener una práctica del ahorro. En términos generales las empresas desarrollan actividades que tienen que ver con el ahorro de forma quincenal y resguardarlo por un periodo de un año. Al finalizar 
este tiempo se entrega a los trabajadores el monto total, iniciando nuevamente la práctica. Siguiendo la teoría de Hofstede, se puede entender que las empresas del destino están desarrollando una de las actividades de sociedades con visión a largo plazo, aunque la visión es sobre un futuro cercano, esto da una sensación de seguridad económica a los colaboradores durante ese periodo de tiempo.

\subsection{Perseverancia}

Una definición de perseverancia menciona que es la tendencia del individuo a comportarse sin ser reforzado en los propósitos de motivación y al no desfallecer en el intento (Cardona, 2000).

La perseverancia es otra de las características que identifican a las organizaciones con visión a largo plazo, ya que se trabaja hoy para obtener beneficios en un futuro, para esta variable las preguntas que se relacionaron, evalúan a consideración de los sujetos de investigación si la gente que trabaja dentro de la empresa lo hace de manera perseverante, o bien si la empresa fomenta esta clase de valores y en caso de hacerlo cómo lo hace. La gráfica siguiente muestra los resultados que se obtuvieron durante el levantamiento de la encuesta.

\section{Gráfico 4. Perseverancia}

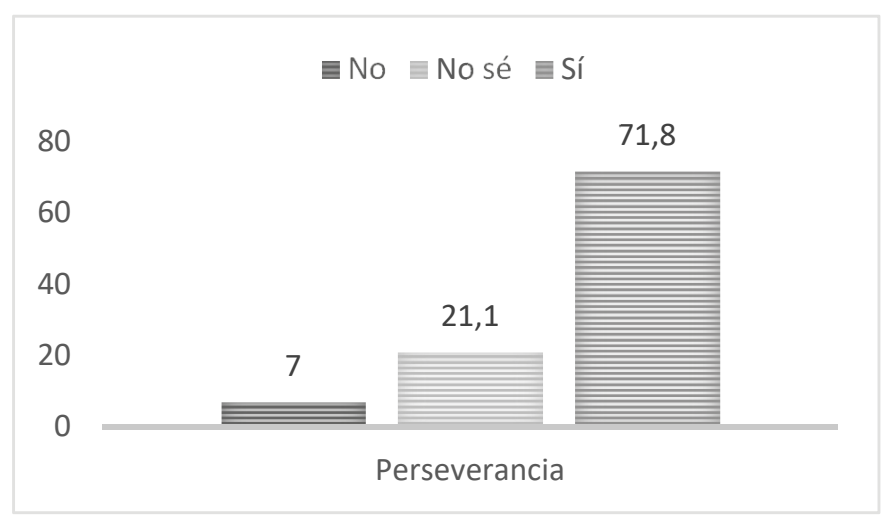

Fuente: elaboración propia con datos de la encuesta (30/04/2016)

La perseverancia es otra de las características que identifican a las organizaciones con visión a largo plazo, ya que se trabaja hoy para obtener beneficios en un futuro. Para este caso en particular este valor atiende a la lucha diaria de los colaboradores dentro de las organizaciones para lograr el cumplimiento de sus objetivos, como lo menciona Cardona (2000) luchar sin desfallecer para lograrlos.

En esta variable el $71.8 \%$ del universo mencionan que efectivamente es la perseverancia un valor que se práctica constantemente en las empresas, sobre todo con el cumplimiento de las metas. Para ello se establecen objetivos claros, específicos y con fecha de cumplimiento, considerando que es una forma de inculcar la perseverancia; es el tener que trabajar duro por 
conseguir lo que se espera en un futuro cercano, ya que esto traerá consigo ciertos beneficios, que en la mayoría de las ocasiones es monetario.

\subsection{Conservación de las tradiciones}

Macías (2012) define a la tradición como la expresión de una actividad que se ha repetido de generación en generación y señala un tipo específico de actividad acompañada de un vocabulario determinado, expresiones corporales, vestuario específico de un escenario participativo, de una fecha, y se convierte en un elemento que aglutina a los grupos humanos; un elemento de participación cultural, sensibilidad y sentimiento de pertenencia.

Hablando de las tradiciones, la región en donde se encuentran las empresas, objeto de este estudio, es rica en tradiciones. Sin embargo, a lo largo de los años, por el desarrollo del CIP y la migración interior en el estado y la república, han hecho que estas tradiciones y cultura se vayan mezclando, creando nuevas y desapareciendo otras.

A pesar de ello para la gente originaria del destino, con raíces de la región, la conservación de estas tradiciones y la cultura sigue siendo algo prioritario en la formación de sus hijos y la familia en general. En ese sentido se desarrolló el siguiente cuestionamiento. ¿Qué tan importante es para usted la conservación de las tradiciones?

Gráfico 5. Conservación de las tradiciones

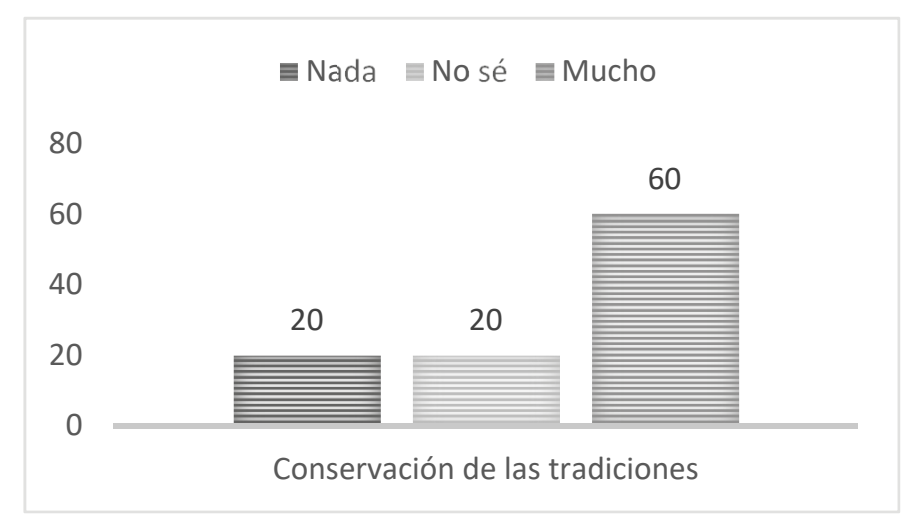

Fuente: elaboración propia con datos de la encuesta (30/04/2016)

El $60 \%$ de los sujetos respondió que era muy importante conservar sus tradiciones y la práctica de las mismas, mientras que para el $20 \%$ realmente esto no es importante, con ello se puede interpretar que se adaptan fácilmente a los cambios y otras culturas.

Es necesario recordar que las tradiciones son parte importante de la identidad de cualquier sociedad, algunas de ellas otorgan mayor importancia a éstas. Es el caso de las sociedades con orientación a corto plazo, ya que ven más al pasado que al futuro con la finalidad de conservar esa identidad que los caracteriza. Para este cuestionamiento se obtuvieron resultados favorables hacia sociedades con orientación a corto plazo. 


\subsection{Obligaciones sociales}

Las obligaciones sociales corresponden a cumplir ciertas actividades con la comunidad en donde se habita o de donde es originaria la persona. Para algunas sociedades esto es verdaderamente un compromiso, característica también de una visión a corto plazo. Las obligaciones sociales también son parte importante de la conservación de las tradiciones, ya que por lo general, el cumplimiento de éstas refuerza los lazos sociales y la identidad de la comunidad. Por el contrario, el incumplimiento de estas obligaciones puede generar conflictos al interior del grupo.

Retomando la definición de Canedo (2008) respecto a los "usos y costumbres", éste puede señalarse desde tres perspectivas distintas pero complementarias: a) lo político, el aspecto más estudiado desde la visión de la elección de los gobernantes de manera tradicional; b) lo económico, partiendo desde los modos en que las sociedades le dan un valor, no necesariamente económico, a los productos y servicios, y las formas de intercambio, como el trueque; c) lo social y d) lo cultural como parte de las obligaciones que cada integrante de la sociedad debe cumplir. El cumplimiento de estas obligaciones sociales, como el dar tequio ${ }^{1}$ por ejemplo, permite el equilibrio y la armonía al interior del grupo. Por el contrario, negarse a cumplir las obligaciones sociales puede, en última instancia, llegar al desconocimiento del individuo como parte de un grupo social.

En el caso del destino en cuestión, como se mencionó anteriormente, se encuentra formado por migrantes de diversos estados de la república, sin embargo, la gente proveniente de comunidades cercanas sí tienen marcada esta contribución social que deben hacer como retribución o agradecimiento a la sociedad, mayormente es el caso de los varones al cumplir la mayoría de edad. En ese sentido, al momento de formar parte de una empresa de servicios turísticos, este compromiso con la sociedad sigue siendo fuerte y se refleja en las respuestas obtenidas, las cuales se muestran a continuación.

\section{Gráfico 6. Obligaciones sociales}

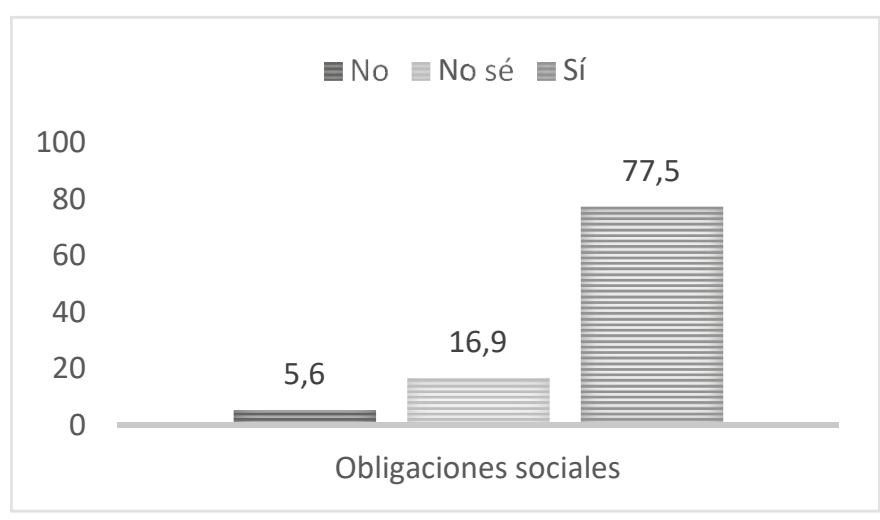

Fuente: elaboración propia con datos de la encuesta (30/04/2017)

1. Entendemos al tequio como una actividad para el bien común. En Oaxaca su uso es frecuente y consiste en un trabajo colectivo, sin pago alguno, para el disfrute de todas y todos. En algunos casos también para el beneficio particular, pero es un favor que debe regresarse en algún momento (reciprocidad). 
Después de preguntar si eran importantes las obligaciones sociales, las repuestas que se obtuvieron fueron, $77.5 \%$ afirmativas y solamente el $5.6 \%$ mencionó no darle importancia. Para lo cual es preciso pensar que esto se refleja en su actuar diario dentro de las empresas para las que trabajan.

Ahora bien, a continuación se muestra una gráfica con todos los resultados obtenidos los cuales apoyaran en la determinación de la caracterización de la cultura organizacional, con base en la propuesta desarrollada por Hofstede, y se podrá identificar la importancia de estas prácticas en el desarrollo de las actividades laborales.

Gráfico 7. Comparativo visión a largo plazo

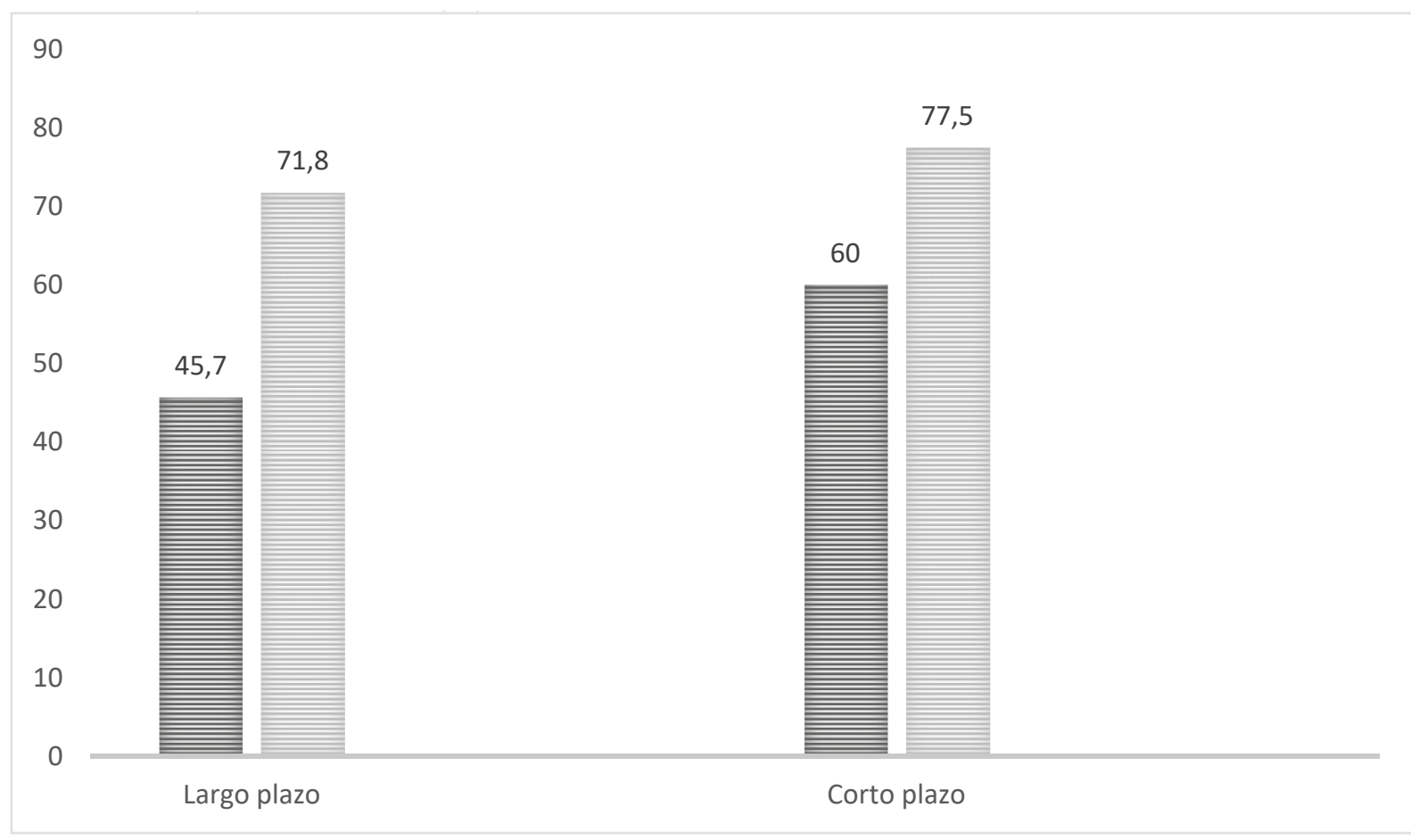

Elaboración propia

La gráfica anterior muestra, en dos bloques, los resultados obtenidos a lo largo de la investigación en donde prevalecen las características a corto plazo, es decir, dentro de las empresas turísticas se tienen colaboradores para los que la perseverancia y el ahorro son ejes fundamentales en su desarrollo laboral, pero al mismo tiempo se tienen colaboradores que marcan a la conservación de las tradiciones y las obligaciones sociales como una característica fuerte en su empresa.

Se ha mencionado que el ahorro y la perseverancia son características que definen a una organización de largo plazo porque estos rasgos tienen una visión hacia el futuro. Sin embargo, estas características no están tan alejadas de las distintas cosmovisiones de los grupos que componen la periferia del CIP y el estado de Oaxaca en general. Como se ha dicho, en la siembra de algún producto se espera tener una cosecha que puede funcionar como 
forma de ahorro durante un tiempo. No obstante, haber logrado lo anterior se traduce en una perseverancia, en una lucha constante sin desfallecimientos para alcanzar el objetivo.

Respecto a la conservación de las tradiciones, éstas resultan ser un componente imprescindible en las cosmovisiones de los colaboradores. Es decir, además de obtener una remuneración por emplear su fuerza laboral dentro de una empresa, los empleados también esperan cierto reconocimiento de sus tradiciones y costumbres en el que se ven reflejados y de los que forman parte.

Las tradiciones, incluso, pueden verse como parte de las obligaciones sociales porque permiten no sólo la conservación de las costumbres y las tradiciones, sino la trascendencia de las mismas. Por ejemplo, mientras que para las empresas turísticas el "día de muertos" (02 de noviembre) en México significa una fecha importante para obtener ganancias, para los colaboradores es casi una obligación social "recordar" a sus fieles difuntos. En torno a este día no sólo está la parafernalia que adorna los altares y los panteones sino la trascendencia de la vida misma en las distintas cosmovisiones mexicanas.

Bajo el contexto anterior se entiende que el cuidado y conservación de la cultura y tradiciones son parte importante y reflejo de la cultura oaxaqueña, la cual es trasladada al interior de las organizaciones y llega a formar parte de la cultura organizacional en donde la empresa aporta nuevo rituales y tradiciones internos, tanto para los colaboradores como para los huéspedes; y por otro lado, los colaboradores aportan ese apego a sus tradiciones y raíces.

En ese sentido la cultura organizacional se ve cada vez más enriquecida, pero al mismo tiempo se convierte en una amalgama con aportaciones de ambas partes que la convierten en única y ésta otorga identidad propia a la organización y a la sociedad misma.

Estos resultados dan pie para seguir realizando investigaciones donde se pueda hacer una correlación para determinar si la importancia que dan los sujetos de estudio hacia el futuro tiene que ver con su origen migratorio, es decir, que provienen de otro estado, mientras que aquellos que dan prioridad al pasado son originarios de la región o bien del estado.

\section{DISCUSIÓN}

Desde el primer estudio hasta sus recientes aportaciones Hofstede ha tenido críticas tanto a favor como en contra. Por ejemplo Fougere y Moulets (2006) mencionan que la segunda edición de Culture Consequences (2001) aporta otra contribución para fortalecer el discurso actual de la gerencia "Cros-cultural" y su idea de la cultura nacional como historia estable.

En ese sentido esta investigación retoma solo una pequeña parte del estudio original, entendiendo que dentro de las organizaciones se tienen una estructura organizacional cros-cultural, lo cual para este caso en particular se conforma de los migrantes del interior de la república y de los colaboradores locales y de aquellas comunidades cercanas.

También se han llevado a la práctica otras investigaciones tomando como referencia la investigación de Hofstede. Por ejemplo, An y Kim (2006) quienes realizaron una comparación de anuncios cibernéticos o la investigación de Milner y Collins (2000) quienes compararon 
anuncios televisivos en diversos países, ambas tienen en común que pretendían identificar las características culturales para con ellos seleccionar la mejor publicidad dependiendo del país en cuestión. Para estas investigaciones el estudio de Hofstede aunque con inconsistencias, es una buena teoría que favorece al estudio de las culturas organizacionales.

Como se observa en los casos anteriores, el estudio de Hofstede favorece la identificación de ciertas características culturales organizacionales, siempre y cuando la metodología y los objetivos del estudio estén perfectamente establecidos. Para este planteamiento en particular se consideró la identificación de cuatro características, las cuales señalan a una cultura con visión a corto o bien a largo plazo.

Existen otros autores que se apoyan en la teoría de Hofstede como Fullagar et al., (2003) quienes opinan que la tipología de Hofstede aún provee un marco provechoso para entender las relaciones de conducta y actitudes en los trabajos, como liderazgo, motivación y estereotipos de género en la gerencia a través de las culturas.

Sin embargo, se tienen también críticas poco favorables al respecto, algunos científicos mencionan que es imposible medir la cultura por dos razones principales, la primera es por la propia naturaleza del concepto "cultura" y la segunda, según Rehner (2009) es que la cultura se crea solamente en el momento de la interacción, por ende no se puede aplicar la idea que existe una cultura observable, medible en forma absoluta, objetiva en un momento y un espacio definido.

De acuerdo con lo mencionado por el autor, se consideró la identificación de las variables culturales a partir de su relación dentro de las empresas turísticas, en el que existe interacción entre los sujetos de estudio, y de acuerdo a su aportación, mientras exista dicha interacción existe cultura, para lo cual se considera valida esta categoría de culturas a corto o largo plazo.

Por otro lado, Mc Sweeney (2002) (citado en Herrera y Bernal, 2013) menciona que es un error tratar la cultura como la única cultura organizacional en IBM, (lugar en donde se realizó el primer estudio de Hofstede), mencionan también que no existe una base de hecho para establecer que las culturas nacionales existen, en las disciplinas de gerencia estratégica solo se justifica este hecho al citar el trabajo de Hofstede (1972). Mientras para Jacob (2005) no existe la pureza cultural, lo que se necesita enfatizar es que los países tienen variedad en culturas y personas.

Con ello, se puede mencionar que efectivamente las aportaciones de Hofstede (1972) y otras más forman parte del marco teórico de esta investigación, tomando en consideración una metodología enfocada hacia un análisis estadístico descriptivo, no probabilístico independiente a la metodología propuesta por Hofstede, con ello se puede llegar a las siguientes conclusiones.

\section{CONCLUSIONES}

Después de revisar los resultados obtenidos y observando las gráficas anteriores se puede interpretar que en el Centro Integralmente Planeado Bahías de Huatulco, las empresas turísticas trabajan con una cultura organizacional enfocada al corto plazo. 
Estos resultados presentan énfasis en la conservación de las tradiciones y el cumplimiento de las obligaciones sociales, lo cual tiene coincidencia con las características de la región. ¿A qué se hace referencia cuando se menciona la coincidencia con la región? Al principio del presente texto se hizo alusión a las distintas formas en que se le da sentido a la vida, a un "sistema cultural" propio de un contexto determinado.

El estado de Oaxaca se caracteriza por las distintas etnias que aquí habitan. Más allá de la lengua materna (en algunos casos el español) y de los rasgos físicos propios de cada grupo, igualmente son particulares las maneras en que se hacen o desarrollan las actividades y las formas de pensamiento. Esta pluralidad de entender el mundo también se manifiesta en otras actividades que se desarrollan fuera del grupo. Los "usos y costumbres" entonces pueden entenderse como elementos imprescindibles para las formas de vida de los grupos sociales.

Aunque el término "usos y costumbres" haga mayor referencia a los sistemas políticos propios de cada lugar, lo cierto es que abarca otros ámbitos de la vida como lo económico, lo social y lo cultural. Para este trabajo se ha delimitado el significado de "usos y costumbres" como aquellas manifestaciones culturales, tanto de pensamiento como maneras de hacer, que trascienden las prácticas cotidianas. Es decir, las formas de hacer o de pensar se trasladan a otros ámbitos, como puede ser el trabajo remunerado.

Para el análisis del presente caso de estudio se observa que los colaboradores de las empresas turísticas en Bahías de Huatulco, les es muy significativo cumplir con las tradiciones y las obligaciones sociales propias de su grupo social. Lo anterior implica que estas personas además de sentirse identificadas, también desean reforzar los lazos sociales con sus congéneres.

Con ello se denota que el CIP Bahías de Huatulco cuenta con colaboradores preocupados por la conservación de sus tradiciones, aun cuando pertenecen a empresas de capital extranjero con formas diferentes de trabajo y por ende con una cultura diferente, esto hace referencia al tipo de planeación de los propios colaboradores, es decir, en términos generales son personas que no visualizan su vida ni trayectoria dentro de la organización a futuro, en todo caso, viven el presente y no preocupan por lo que vendrá después es decir, son colaboradores que viven el momento.

Una característica de las sociedades a largo plazo es que cuentan con planes y metas, por ello trabajan en el presente para poder obtener esos beneficios esperados a futuro, sin embargo, la cultura a corto plazo no funciona de esa manera. Por ello los colaboradores del destino turístico no tienen metas o planes establecido a futuro que rija su comportamiento en la actualidad. Más bien, tienen miedo y resistencia al cambio, les preocupa pensar en lo que vendrá después y correr riesgos no es una alternativa para ellos.

Con lo anterior se puede deducir que los colaboradores del caso de estudio están enfocados al pasado y no tienen una visión a futuro que les ayude a trabajar hoy para obtener beneficios más adelante. En ese sentido las empresas y la sociedad en general, son un reflejo de esta forma de trabajo, por lo que se debería de considerar un cambio, en donde sin restarle importancia a sus tradiciones y obligaciones sociales, se comience a visualizar el futuro como una manera de crecimiento y desarrollo. 
A lo largo de esta investigación se tuvo ciertas limitaciones, con respecto a la resistencia de los colaboradores para responder a los cuestionamientos, sobre todo al pensar que podrían tener consecuencias negativas dentro de sus empresas. En todo caso las respuestas obtenidas muestran la percepción de los mismos a partir de sus experiencias personales, por lo cual esto puede ser un tanto subjetivo. Por ello a partir de esta investigación se puede dar continuidad a este estudio e inclusive desarrollar nuevas líneas de investigación que muestren los procesos de socialización de los colaboradores de las empresas turísticas a partir de sus principales elementos culturales (valores, creencias, ceremonias, etc.) y el fenómeno de la migración interna de los colaboradores con la repercusión cultural que esta trae consigo.

\section{REFERENCIAS}

An, D. y Kim, S. (2006). Relating Hofstede masculinity dimension to gender role portrayals in advertising. International marketing Review, 24(2), 181-207.

Becker, G. (1964). Human capital: a theoretical and empirical analysis, with special reference to education. Nueva York: Columbia University Press.

Becker, G. (2002). La economía cotidiana. México: Planeta.

Canedo, G. (2008). Una conquista indígena. Reconocimiento de municipios por "usos y costumbres" en Oaxaca (México). En A.D. Cimadamore (comp.), La economía política de la pobreza (pp. 401-426). Buenos Aires: CLACSO.

Cardona, A. (2000). Formación de valores: teoría, reflexiones y respuestas. México: Grijalbo, Universidad Autónoma de Baja California.

Chamorro, E. (2016). Las dimensiones culturales de Geert Hofstede y la intención emprendedora en estudiantes universitarios del departamento del Quindío (Colombia). Pensamiento \& Gestión, 41, 60-90.

Comisión Nacional de Áreas Protegidas (CONANP). (2003). Programa de Manejo Parque Nacional Huatulco México: CONANP.

Cotler, P. (2003). Las entidades microfinancieras del México urbano. México: Universidad Iberoamericana, A.C.

Fang, T. (2003). A critique of Hofstede fifth national culture dimension. International Journal of Cross Cultural Management, 3, 347-368.

Fondo Nacional de Fomento al Turismo (FONATUR). Página web [www.fonatur.gob.mx]. Consultado el 01 de Febrero de 2017

Fougère, M. y Moulettes, A. (2006). Development and modernity in Hofstede's culture's consequences: a postcolonial reading. Lund Institute of Economic Research: Working Paper, 2(1) 1-16.

Fullagar, C., Sumer, H., Sverke, M., y Slick, R. (2003). Managerial sex-role stereotyping: a cross cultural analysis. International Journal of Cross Cultural Management, 3(1), 93-107.

García, C. (2004). Diferentes, desiguales y desconectados. Mapas de la interculturalidad. Barcelona: Gedisa.

Guillen, J. (2007). Administración de proyectos con equipos multiculturales y geográficamente dispersos (Aspectos humanos y comunicaciones). (Tesis doctoral). Universidad para la Cooperación Internacional. San José, costa Rica. 
Hernán, M. (2006). La cultura y el turismo como medios de desarrollo socioeconómico. Ponencia presentada en el Seminario Iberoamericano de Cultura, Turismo y Desarrollo (AECl-acerca/OEI), Cartagena de Indias, Colombia. Disponible en http://www.oei.es/ historico/cultura/culturamhmejia.htm Consultado el 15 de Enero de 2018.

Herrasti, L. (2013). Distintos modos culturales de organizar el trabajo orientación a corto o largo plazo. Relaciones laborales y recursos humanos. 3, 62-87.

Herrera, J. y Bernal, D. (2013). Sobre la transferencia de modelos organizacionales. Gestión y política pública, 23(2), 331-357.

Hofstede, G. (1972). Private correspondence. Estados Unidos: Sage.

Hofstede, G. (1984). Culture's consequences. International differences in work-related values. Estados Unidos: SAGE.

Hofstede, G. (2001). Culture's consequences: Comparing values, behaviors, institutions, and organizations across nations. Thousand oaks. California: Sage Publications.

Jacob, N. (2005). Cross cultural Investigations: Emerging Concepts. Journal of Organizational Change Management, 18, 514-528.

Lev, B. (2004). Intangibles en la encrucijada. Revista de contabilidad y dirección, 1, 15-29.

Macías, R. (2012). El trabajo sociocultural comunitario. Fundamentos epistemológicos, metodológicos y prácticos para su realización. España: Universidad de Málaga.

Méndez, C. (2003). Clima Organizacional en Colombia. El IMCOC: un método de análisis para su intervención. Colombia: Centro editorial Universidad del Rosario.

Mendoza, M., Monterrubio, J., Fernández, M. (2011). Impactos sociales del turismo en el Centro Integralmente Planeado (CIP) Bahías de Huatulco, México. Gestión turística, 15, 47-63.

Milner, L. y Collins, J. (2000). Sex-roles portrayals and the gender of nations. Journal of Advertising, 29(1), 67-79.

Mooij, M. y Hofstede, G. (2010). The Hofstede model. Applications to global branding and advertising strategy and research. International Journal of Advertising. 29(1), 85-110.

Navarro, I. (2005). Capital humano: su definición y alcances en el desarrollo local y regional. Archivos analíticos de políticas educativas. 35 (13), 2-37.

Rehner, J. (2009). Nation und kultur. In H. -D Haas, \& S. - M. Neumair. Internationale Wirstschaft. (pp. 349-375). München: Oldenbourg.

Rozas, P. y Sánchez, R. (2004). Desarrollo de infraestructura y crecimiento económico: revisión conceptual. Santiago de Chile: CEPAL.

Salazar, C. y Ramírez, J. (2003). Open regionalism: a great vision of the Mexican Pacific. Colima, Col., México: Foro Académico del Pacífico Mexicano.

Sampieri, R., Fernández, C. y Baptista, P. (2006). Metodología de la Investigación. México: McGrawHill.

Schultz, T. (1961). Investment in human capital. American Economic Review. 51 (1) 1-17.

Talledos, E. (2012). La transformación del espacio y los usos del agua en San Miguel del Puerto, Pochutla, Oaxaca. (Tesis doctoral) Universidad Nacional Autónoma de México.

Vera, J. y Rodríguez, K. (2009). La psicología social y el concepto de cultura. En Psicología \& Sociedad. 21, 100-107.

Vialadot, M. (2008). Direcció estratégica de sistemes i tecnologies de la información. España: UOC. 